\section{A teorização sobre processo de trabalho em saúde como instrumental para análise do trabalho no Programa Saúde da Família}

\author{
Theoretical review of the work process \\ in health care used to analyze work \\ in the Family Health Program in Brazil
}

Edilza Maria Ribeiro ${ }^{1}$

Denise Pires 1

Vera Lúcia G. Blank 2

\footnotetext{
1 Departamento

de Enfermagem,

Centro de Ciências da Saúde,

Universidade Federal

de Santa Catarina,

Florianópolis, Brasil.

2 Departamento de Saúde

Pública, Centro de Ciências

da Saúde, Universidade

Federal de Santa Catarina,

Florianópolis, Brasil.

Correspondência Edilza Maria Ribeiro Departamento

de Enfermagem,

Centro de Ciências da Saúde,

Universidade Federal

de Santa Catarina.

Campus Universitário

Trindade, Florianópolis, SC

88040-970, Brasil.

edilzamr@linhalivre.net
}

\begin{abstract}
This literature review focused on the work process in health care,particularly issues linked to the Family Health Program. Since 1994, the Program has rapidly expanded health coverage for the Brazilian population, and as a result the Program's work force has also increased. The Program thus requires attention, allocation of responsibilities, and contributions of various types by stakeholders and institutions. The work occurs in the services sector, within a neoliberal scenario. Work in health care shares some characteristics with other occupations in the services sector, such as bureaucratization, the influence of capitalist division of labor, and difficulties in teamwork, in addition to little regard for the subjectivity of health system clients and workers. The study particularly focuses on work conditions of family health teams in Brazil,including strategies for revitalizing health care work and coping with alienating conditions in the work process. Finally, the study reiterates the dialectic condition of the work process in health care under the Family Health Program, with its possibilities for success, conceived and functioning in the midst of contradictions and difficulties.
\end{abstract}

Family Health Program; Working Conditions; Workers

\section{Introdução}

O Programa Saúde da Família (PSF) foi formulado pelo Ministério da Saúde do Brasil (MS) no ano de 1993, e no final do ano de 2002 já estava implantado em 4.114 municípios brasileiros. Conta com 16.192 equipes de saúde da família e faz a cobertura de 53 milhões de pessoas 1 .

Em virtude da importância que o PSF adquire por sua expansão e difusão, pelo seu potencial de inclusão de parcelas crescentes da população brasileira na assistência em saúde e pelo considerável contingente de trabalhadores envolvidos, busca-se, neste texto, refletir sobre o trabalho das equipes do programa, utilizando-se como referência a teorização sobre o processo de trabalho em saúde.

\section{O trabalho no setor de serviços e o trabalho em saúde}

Os estudos de Braverman 2, Mills 3, Offe 4 e Pires 5,6,7 contribuem para o entendimento do processo de trabalho em saúde como parte do setor de serviços com o qual tem identidade, mas também tem especificidades que estimulam estudos em profundidade. $\mathrm{O}$ trabalho em saúde é hoje, majoritariamente, um trabalho coletivo institucional, que se desenvolve com características do trabalho profissional e, também, da divisão parcelar ou pormenorizada do 
trabalho e da lógica taylorista de organização e gestão do trabalho.

Diversos autores têm apontado que o conjunto de atividades que não estão diretamente envolvidas na produção industrial e que não estão no setor primário da economia tem crescido, progressivamente, desde a Revolução Industrial. Esse conjunto de atividades constitui o chamado setor de serviços, e muitas delas, que eram feitas no âmbito da empresa, passaram a ser desenvolvidas por serviços especializados fora dela. Da mesma forma, cresceu o conjunto de atividades comerciais, de serviços de educação, de saúde, de segurança pública, dentre outros.

No início do século XX, os resultados da aplicação dos princípios da gerência científica, baseados nas formulações de Taylor, começam a ser conhecidos e ampliados de forma crescente, influenciando não só o trabalho industrial, mas também o trabalho no setor de serviços. Surgem estudos sobre o tempo de execução das tarefas, registros das quantidades de trabalho desempenhado, rotinizações, reorganização física dos ambientes de trabalho para diminuição dos tempos gastos sem produção, etc. Para aumentar a produtividade, expande-se a divisão parcelar do trabalho e a mecanização da produção 2 .

Com o aumento do fluxo de trabalho e a aplicação de novos métodos de gerência, o processo é dividido em operações mínimas, e os trabalhadores perdem a compreensão da totalidade do processo de trabalho. O tempo empregado na atividade está sob constante exame e controle. $\mathrm{O}$ trabalho pensante fica restrito a um pequeno grupo, esperando-se da maioria a execução das atividades delegadas de cunho manual 2.

Braverman 2 participa do debate sobre as características da sociedade pós-Segunda Guerra Mundial e defende a tese de que, apesar do grande crescimento dos setores não envolvidos diretamente com a produção material, a tendência não é de ampliação de uma outra racionalidade diferente da encontrada no trabalho industrial. Diz que este grupo, apesar de apresentar certa diferenciação salarial que lhe permite um afastamento dos piores aspectos da situação do proletariado, "não possui qualquer independência econômica ou ocupacional; é empregado pelo capital e afiliados; não possui acesso algum ao processo de trabalho ou meios de produção fora do emprego e deve renovar seus trabalhos para o capital incessantemente a fim de subsistir" 2 (p. 341).
Sua atuação faz aumentar o capital e, nesta relação, embora predomine a subordinação à autoridade e submissão à exploração, esses trabalhadores tanto têm prerrogativas e privilégios do capital, como comungam características da condição proletária, avançando, porém, na direção do proletariado 2 .

Para Braverman 2, essas "camadas médias de emprego" incluem, dentre outros, trabalhadores especializados como engenheiros, enfermeiros, técnicos, empregados de vendas, da administração financeira e organizacional e assemelhados. Os trabalhadores deste extrato estão submetidos a variadas condições de trabalho e o excedente de mão-de-obra permite não só a diminuição dos salários, como também a piora das condições de trabalho.

Mills 3, ao estudar a realidade americana dos anos 50, diz que os profissionais liberais foram os que mais se modificaram na passagem da antiga para a nova classe média. Muitos deixaram de exercer suas atividades de forma independente e passaram à condição de empregados. De possuidores de amplo conhecimento transformaram-se em especialistas e suas atividades fragmentaram-se, passando a requerer complementaridade de atividades parcelares e rotineiras de outros grupos de trabalhadores. No setor saúde, os médicos, majoritariamente, ocupam espaços de decisão e mantêm certa independência no que diz respeito ao seu trabalho, e poucos dependem unicamente do assalariamento. No que diz respeito aos trabalhadores de enfermagem, a maioria é assalariada e apenas uma pequena parcela de enfermeiros atua de forma independente, fora do trabalho coletivo institucional.

Para Offe 4 , as sociedades ocidentais, a partir das últimas décadas do século XX, têm sido chamadas de sociedades de serviços, dado o importante aumento destes. Uma característica distintiva dos serviços é a dificuldade de planejamento em relação ao custo/beneficio, uma vez que muitos deles necessitam estar à disposição da sociedade e há incerteza de quando as necessidades serão demandadas, como é o caso dos serviços de atenção à saúde, dos serviços de proteção e os de consumo em redes comerciais. Há incerteza em relação ao volume, ao tipo, ao momento e em relação às necessidades do cliente. Isso faz com que o planejamento conte com dados menos precisos do que na produção material. Esse mesmo autor destaca ainda que, diferentemente do setor da produção material industrial, onde produ- 
tos não rentáveis podem deixar de ser fabricados, muitos serviços não podem parar, mesmo instituições que não são rentáveis economicamente, como é o caso dos serviços de saúde.

\section{O processo de trabalho em saúde na conjuntura atual}

Estudos de autores ligados à saúde coletiva, tais como os de Campos 8, Merhy 9, Pires 7, Sá 10, Schaiber 11, Soares 12, Almeida 13, entre outros, têm possibilitado o diálogo, que vem sendo desenvolvido no PSF, entre a teorização sobre "processo de trabalho em saúde" e realidade do trabalho.

Segundo Pires 7 (p. 85), o "trabalho em saúde é um trabalho essencial para a vida humana e é parte do setor de serviços. É um trabalho da esfera da produção não material, que se completa no ato de sua realização. Não tem como resultado um produto material, independente do processo de produção e comercializável no mercado. O produto é indissociável do processo que o produz; é a própria realização da atividade".

Aponta, também, que hoje, em sua maioria, esse trabalho é coletivo, realizado por diversos profissionais de saúde e diversos outros grupos trabalhadores que desenvolvem uma série de atividades necessárias para a manutenção da estrutura institucional. Envolve características do trabalho assalariado e da divisão parcelar do trabalho, bem como características do trabalho profissional do tipo artesanal.

Algumas categorias profissionais executam o seu trabalho aplicando características da divisão parcelar do trabalho, como, por exemplo, a enfermagem e a farmácia-bioquímica, nas quais se encontra a fragmentação de tarefas sob o controle gerencial dos profissionais de nível superior.

"O trabalho é compartimentalizado, cada grupo profissional se organiza e presta parte da assistência de saúde separado dos demais, muitas vezes duplicando esforços e até tomando atitudes contraditórias. Os profissionais envolvidos dominam os conhecimentos para o exercício das atividades específicas de sua qualificação profissional, no entanto, os médicos, no âmbito do trabalho coletivo institucional, ao mesmo tempo que dominam o processo de trabalho em saúde, delegam campos de atividades a outros profissionais de saúde como enfermagem, nutrição, fisioterapia,etc. Esses profissionais executam atividades delegadas mas mantêm certo espaço de decisão e domínio de conhecimentos, típico do trabalho profissional" 7 (p. 89).
Considerando a teorização de Offe 4 sobre as alternativas de racionalização empreendidas quando algum serviço se torna inviável economicamente, Pires aponta que, nos serviços de saúde, diversas saídas são possíveis como: a introdução de inovações tecnológico-organizacionais; a redução da força de trabalho; a redução da força de trabalho qualificada; a redução de salários; a precarização das condições de trabalho; a externalização, o auto-serviço, dentre outras. "A implementação de uma ou outra medida, ou de um conjunto delas, depende de diversos fatores, especialmente da capacidade dos diversos grupos e classes sociais fazerem valer os seus interesses" 14 (p. 256).

Quando essas medidas não são suficientes, no caso dos serviços privados, os serviços podem simplesmente fechar, como ocorre nas empresas capitalistas. Já no caso dos serviços públicos, dá-se a interferência de um complexo de fatores macropolíticos na escolha da estratégia de racionalização a ser empregada em cada momento histórico. Além disso, em situações que envolvem altos custos, a decisão de fechar o serviço torna-se mais difícil se envolver necessidades humanas básicas.

Segundo Soares 12, o setor saúde, no Brasil, em especial na década de 90, tem sido afetado pelas ações governamentais decorrentes da política de "Reforma do Estado", apontada como necessária para o processo de modernização e inserção do País de forma competitiva no cenário internacional. A proposta de reforma defendeu a substituição do modelo administrativo das instituições públicas, localizando na questão gerencial o nó crítico do problema das fragilidades dos serviços públicos de saúde. E os problemas de fundo que geram a desigualdade no País permanecem, dentre eles o padrão de financiamento do Estado, sua incapacidade de produzir políticas de distribuição de renda e equalização do acesso a serviços públicos essenciais.

O modelo assistencial, vigente nos serviços de saúde, guia-se pela ótica hegemônica neoliberal e, na prática, essa visão acaba definindo a missão dos serviços e as conformações tecnológicas, atendendo a interesses poderosos, considerados legítimos 9

Nesse modelo, segundo Schraiber et al. 11 (p. 239), "há a tendência de se adotar políticas que passem tanto a desproteger o trabalho e o trabalhador, quanto, no caso da saúde, a regular diretamente o produtor direto dos cuidados, interferindo imediatamente em seus processos de trabalho".

Campos 8 aponta para a fragmentação, a imobilidade, a desarticulação das linhas de in- 
tervenção com os sistemas de informação e para a burocratização, como problemas importantes no trabalho de saúde pública brasileiro. Para o autor, o trabalho das equipes sofre influência da linha taylorista, traduzida pela acumulação de normas administrativas e padronizações técnicas, guiadas por crenças na possibilidade de controlar e regulamentar o conjunto total do trabalho.

Também ocorrem dificuldades entre a proposta de cuidado/assistência generalista e a baseada na especialização. No primeiro caso, haveria o risco da transformação das profissões e dos especialistas em técnicos polivalentes, com atribuições semelhantes, perdendo, na prática, a especificidade. De outro lado, a especialidade encarregada de uma parte da intervenção não poderia ser responsabilizada pelo resultado global do tratamento. Os resultados desse modelo, baseado na especialidade e na diminuição crescente da capacidade de cada profissional resolver problemas, têm sido tratamentos cada vez mais longos, envolvendo inúmeros especialistas, com custos crescentes, com mais sofrimento e restrições impostas aos clientes e uma perda da visão integral do sujeito que necessita de cuidados em saúde 8 .

Os compromissos passaram a ser indiretos, com a saúde em geral, com a população, com o saber, com a coletividade, distanciando-se dos sujeitos/usuários clientes ou parcela real da população 8 .

A indicação da retomada de generalistas e da constituição de equipes multiprofissionais tem sido recomendada, colocando-se sobre estas conformações inúmeras expectativas. Nas palavras de Campos, "imagina-se que estas equipes milagrosamente compensariam a desresponsabilização e a fragmentação que a especialização excessiva cria, autoriza e legitimiza" 8 (p. 247).

Para Schraiber et al. 11, a divisão do trabalho na área da saúde gerou a condição de complementaridade e interdependência dos diversos trabalhos especializados. Quando a equipe é multiprofissional e os agentes possuem autoridades desiguais, a tensão surge do embate entre a complementaridade e interdependência e busca da ampliação da autonomia técnica dos profissionais. Todavia, o requerido para a eficácia e eficiência dos serviços seria a autonomia técnica aliada à articulação de ações.

Mattos 15 aponta como um dos problemas do trabalho em saúde dificuldades na passagem do político para o assistencial, que se expressam na identidade dos atores sociais e nos seus projetos. Para o autor, os agentes costumam identificar-se mais pelas suas inserções no processo de trabalho do que pelas suas inserções político-ideológicas. Esta situação impõe desafios para a gestão do trabalho cotidiano das equipes de trabalho enquanto (re)criação de projetos coletivamente sustentados que necessitam estar associados à (re)criação de novos sujeitos sociais defensores de certas crenças e valores.

\section{O trabalho no Programa Saúde da Família}

Questionamentos quanto a ser um programa ou estratégia compõem uma das controvérsias envolvendo o PSF. Lançado como programa em 1994, foi catalogado como estratégia, buscando diferenciar-se dos programas tradicionais já produzidos pelo MS. A segunda versão oficial do PSF, publicada em 1997, define-o efetivamente como estratégia de reorientação do modelo assistencial. Sua implantação segue abrigando tal dicotomia: de um lado, ratifica sua condição de programa que se apresenta com objetivos, metas e passos definidos, ou seja, com uma formulação e com modus operandi estabelecido/normatizado; de outro lado, reivindica a posição de "estratégia” por sinalizar um caminho possível para se atingirem os objetivos do SUS, reorientando o modelo assistencial a partir da atenção básica, sendo, portanto, capaz de influenciar e produzir impactos no sistema como um todo.

As equipes de saúde da família, formadas basicamente pelo médico generalista ou de família, enfermeiro, auxiliar de enfermagem e agentes comunitários de saúde, têm como objetivos centrais a prestação de assistência integral, contínua, com resolutividade e qualidade, às necessidades de saúde da população adscrita, destacando-se a perspectiva da família. $\mathrm{Pa}-$ ra atingir tais objetivos, requer-se abordagem multidisciplinar, processos diagnósticos de realidade, planejamento das ações e organização horizontal do trabalho, compartilhamento do processo decisório, além do estímulo ao exercício do controle social 16.

Essa proposta constitui-se em um importante desafio, já que se propõe a uma ruptura com o modelo assistencial atual e à construção de uma nova prática, com uma nova dimensão ética. Ademais, esse desafio está em curso em um cenário profundamente influenciado pelo modelo biomédico, seja na formação profissional, seja na prática assistencial hegemônica.

Alguns estudos realizados com base na realidade cotidiana das equipes do PSF mostram essa prática como possuidora de um potencial 
positivo de mudança; outros, porém, mostram que existem grandes limitações para que isto ocorra.

Bertoncini 17 , por meio de pesquisa efetuada com profissionais das equipes de saúde da família em Blumenau, Santa Catarina, identifica mudanças e possibilidades de ocorrer um diferencial entre as práticas anteriores e aquelas decorrentes da proposta do PSF, reforçando os autores que apontam o potencial positivo do programa. As equipes citam como mudanças em seu trabalho atuarem de forma comprometida, baseada no vínculo e co-responsabilidade; contarem com maior adesão da população; desenvolverem um trabalho diferenciado dos outros serviços de saúde pública; produzirem ampliação das ações de promoção; terem a possibilidade de desenvolver assistência integral e contínua voltada para a população/ comunidade; reduzirem as internações hospitalares; produzirem melhora do acesso da população à rede básica de saúde; efetuarem o diagnóstico das doenças mais precocemente; introduzirem práticas assistenciais inovadoras; incrementarem a utilização de práticas assistenciais de baixa aplicação no modelo anterior.

Sousa 18 também aponta para inúmeros impactos evidenciados em vários municípios ao longo da implantação do PSF, tais como: "alto nível de satisfação da população com o atendimento das equipes; melhoria da vigilância à saúde da população; utilização adequada dos serviços de maior complexidade com redução das internações hospitalares desnecessárias; maior qualidade, cuidado com a atenção prestada;elevação da resolutividade das redes assistenciais básicas (que passou a girar em torno de 90\%)" 18 (p. 50). Esses impactos se constituíram fatores motivadores da disseminação do programa pelo País.

Destaque-se, porém, que uma série de condições envolvendo o processo de trabalho das equipes do PSF dificultam, deterioram e tornam vulnerável tal processo.

Soares 12, analisando o Programa de Agentes Comunitários de Saúde (PACS) e o PSF, chamados carros-chefes da mudança do modelo assistencial, mostra as dificuldades para que isso ocorra: (1) a imposição dos programas pelo MS; (2) o fato de serem totalmente normatizados no nível central; (3) o fato de serem financiados por incentivos financeiros internacionais; (4) a obrigatoriedade, na prática, de sua adoção pelos municípios; (5) a desconsideração da enorme heterogeneidade dos municípios, na oferta de serviços de saúde; (6) ausências de redes regionalizadas e hierarquizadas para garantir o acesso universal a todos os ní- veis de atenção - o que limita o atendimento dado por esta estratégia a uma simples porta de entrada sem saída; (7) a desconsideração da complexidade do quadro epidemiológico brasileiro; e, finalmente, a substituição de profissionais qualificados da Enfermagem por pessoas sem qualificação, com contratos precários de trabalho, com sérias limitações na resolução dos problemas de saúde da população.

Mattos 15, também referindo-se a essa questão, entende que a descentralização implementada no âmbito do SUS gerou uma multiplicidade de sistemas municipais de saúde, diferentes entre si quanto à participação do setor privado, na provisão de serviços ao SUS, nas configurações de rede adotada em cada sistema, o que influi enormemente nos modelos gerenciais e na sua diversidade.

Fertonani 19, estudando o trabalho de equipes do PSF de Maringá, Paraná, encontrou que o modelo é centrado no médico e no atendimento curativo; que o trabalho dos profissionais envolvidos mantém as características de compartimentalização, com execução de tarefas prescritas, sem um planejamento coletivo aderente às necessidades da população da sua área de abrangência; que as atividades desenvolvidas no domicílio não representam uma ruptura com o modelo biomédico.

Pesquisas como as de Bertoncini 17, Costa et al. 20,21, Souza 22, têm exposto as condições de trabalho em que as equipes estão imersas, destacando a disponibilidade de recursos humanos, as formas de contrato, a infra-estrutura material, a dinâmica da assistência e condições sócio políticas para desenvolvimento do trabalho. Assim, os autores citados apontam para composição básica insuficiente das equipes; insuficiência de profissionais com o perfil proposto pelo programa; várias modalidades de contrato de trabalho; heterogeneidade na estrutura física das unidades de saúde da família, estando algumas inadequadas e em situação precária; sobrecarga de atendimento, gerando dificuldades em efetuar o planejamento e discutir a dinâmica do trabalho; comprometimento do fluxo e contrafluxo dos usuários e informações entre os diferentes níveis do sistema; problemas de fidedignidade no preenchimento do Sistema de Informação de Atenção Básica (SIAB); diferentes estilos de gestão das equipes de saúde da família, configurando relações ora aproximativas, ora conflituosas; expectativas contraditórias e conflitos das equipes de saúde da família com os poderes locais; conflitos na relação entre o PSF e a população, quando as equipes não conseguem atender a demanda. 
Franco \& Merhy 23 apontam problemas com o alto grau de normatividade na implementação do PSF. O formato das equipes, as funções de cada profissional, o cadastro das famílias, o levantamento dos problemas de saúde existentes no território e os modos de fazer o programa são regulados pelo MS. O caráter prescritivo do PSF é exacerbado. São definidos a priori os locais de assistência e a lista de atividades a serem realizadas por equipe, confundindo-se o que são ferramentas de diagnóstico e intervenção com o que é resultado em saúde: “(...) infere-se que seguindo a prescrição altamente detalhada obter-se-á o resultado anunciado" 23 (p. 7).

Para Bertoncini 17, as condições de trabalho apontadas produzem alienação, impotência, estresse, conflitos, disputa por poder e sentimentos de medo, insegurança, baixa auto-estima, dificultando, assim, as iniciativas para mudar as condições vigentes e garantir a integralidade da assistência.

\section{Considerações sobre o processo de trabalho no PSF e alternativas para a modificação das condições de trabalho}

O trabalho no PSF pretende, segundo seus defensores, constituir-se em uma "estratégia estruturante" de um novo modelo assistencial em saúde. No entanto, os estudos sobre a realidade atual, após nove anos de implementação do PSF no Brasil, mostram que existem tanto pontos positivos na proposta, em direção à ruptura com a lógica taylorista de organização e gestão do trabalho, quanto problemas em relação a condições de trabalho. Além disso, verifica-se a persistência dessas características no trabalho em saúde e, também, no trabalho desenvolvido no PSF.

Uma das características do trabalho no setor de serviços apontadas por Offe 4 é requerer uma forma mais flexível de planejamento, em virtude da imprevisibilidade da demanda, do momento de consumo e do tipo de serviço que será necessário. Essa característica torna mais complexa a questão do financiamento em saúde, porque, mesmo em condições ideais, de não-desperdício e de alta competência técnico-gerencial, é preciso considerar a necessidade de "estar disponível para”, como para as situações de emergência ou para desenvolver ações de custo elevado. Essas situações não podem ser submetidas, unicamente, a critérios econômicos.

Outra alternativa para a racionalização dos custos, apontada por Offe 4 , e que está presen- te no PSF é a precarização do trabalho, como se pode verificar pela flexibilização das formas de contratação das equipes e contratação de trabalhadores sem qualificação profissional para o exercício de ações de saúde, que é o caso dos ACS

Campos 8 aponta alguns eixos operativos úteis para potencializar o trabalho em saúde, tais como o jogo entre autonomia e responsabilidade, gestão participativa e mudança de aspectos da clínica e da saúde pública. A posição em favor de uma radical autonomia de profissionais e instituições é defendida por vertentes da administração de recursos humanos em saúde, que têm no pagamento por produção o principal mecanismo regulador do trabalho. Segundo o mesmo autor, esse mecanismo pode aumentar a produtividade, mas não dá conta de articular trabalho e compromissos sólidos com a produção da saúde. "A capacidade de se definir e cobrar responsabilidades destes modelos de gestão é portanto muito pequena" 8 (p. 231). Terceirização do atendimento a clientes, compra de serviços de cooperativas, pagamentos por produção ou lógicas baseadas na produtividade geram superprodução de atos desnecessários mas rentosos, em detrimento dos necessários e pouco remunerados.

Campos 8 mostra, ainda, que podem ser tentados arranjos institucionais que atendam pelo menos a três critérios, combinando autonomia e responsabilidade atribuída aos profissionais. Esses critérios às vezes são antagônicos entre si, mas se constituem em aspectos gerenciais importantes. O principal critério para validar um arranjo institucional é a capacidade de "produzir saúde" do serviço ou da equipe; um outro critério seria a viabilidade técnica, financeira e política dos projetos ou arranjos acordados (dinheiro, armação político-institucional, saberes e meios); o terceiro consideraria realização profissional e financeira dos trabalhadores da saúde. Do ponto de vista prático, seria desejável buscar-se sempre a máxima realização possível dos três critérios 8 .

Para Campos 8 (p. 235), “(...) a recuperação da prática clínica assentada no vínculo é a maneira prática de se combinar autonomia e responsabilidade profissional". Organizar o atendimento clínico com base no vínculo permitiria que as instituições acompanhassem e identificassem os resultados do trabalho de cada profissional e ainda fomentaria o exercício da cidadania pelo paciente.

Ainda segundo Campos 8, "é necessário reaproximar os trabalhadores do resultado de seu trabalho" (p. 235). Isto pode ser favorecido pela utilização de mecanismos que permitam o 
envolvimento/participação em novas formas de funcionamento institucional, na reinvenção como possibilidade cotidiana, comprometendo trabalhadores com a missão e os projetos institucionais. Segundo Sá 10, o cumprimento ou operacionalização de princípios como universalidade e integralidade da assistência, participação popular e profissional, qualidade técnico-científica, depende grandemente da "possibilidade de abertura ou desencadeamento de processos sociais e intersubjetivos de criação/recriação constante de acordos, pactos, projetos coletivos, sempre conjunturais e transitórios, que representem a eterna busca do ideal (necessária à vida, entendemos) mas sem cair na doença idealidade" 10 (p. 256).

Também adquire centralidade o desenvolvimento, a criação de novos indicadores, avaliadores do desempenho como possibilidade de dimensionar a retribuição financeira dos trabalhadores de saúde. Uma fórmula com melhores resultados para alterar positivamente a subjetividade desses trabalhadores (diminuição de conflitos, aumento do envolvimento com o projeto de saúde, sentimento de valorização por sua contribuição) seria combinar salários fixos com outras variáveis de pagamento, como gratificação pelo alcance de metas 8 .

Azevedo et al. 24 , nessa mesma linha de proposições, ao analisaram novos processos de gestão, como os da qualidade total e o enfoque estratégico de planejamento, surgidos como respostas à crise de governabilidade do sistema e das organizações de saúde, enfatizam que é preciso ir adiante a fim de ultrapassar concepções também limitantes, presentes nestes enfoques. Devem-se superar concepções do indivíduo como criatura meramente funcional, não considerado como sujeito político e como pessoa singular, provido de crenças, valores, desejos e emoções, no caso da gestão da qualidade total. No caso do enfoque estratégico, é necessário superar a ênfase exacerbada na racionalidade político-estratégica, que ignora uma subjetividade mais ampla, restringindo-a à subjetividade elaborada na consciência (racional). “(...) Impõe-se a necessidade da revisão da própria concepção de organização, como da noção de sujeito e da compreensão dos processos intersubjetivos em seu interior" 24 (p. 240).

A expressão multidimensional das necessidades de saúde, sejam elas individuais ou coletivas, o conhecimento sobre o complexo objeto e as intervenções nele - processo saúde-doença de indivíduos e/ou grupos, requerem múltiplos sujeitos para darem conta da totalidade das ações, demandando a recomposição dos trabalhos especializados, com vistas à assistên- cia integral. A mera alocação de recursos humanos de diferentes áreas não garante tal recomposição; ações isoladas, ações justapostas, sem articulação, não permitem o alcance da eficácia e eficiência dos serviços na atenção à saúde 11 .

Trabalho em equipe de modo integrado significa conectar diferentes processos de trabalhos envolvidos, com base em um certo conhecimento acerca do trabalho do outro e valorizando a participação deste na produção de cuidados; é construir consensos quanto aos objetivos e resultados a serem alcançados pelo conjunto dos profissionais, bem como quanto à maneira mais adequada de atingi-los.

Significa também utilizar-se da interação entre os agentes envolvidos, com a busca do entendimento e do reconhecimento recíproco de autoridades e saberes e da autonomia técnica.

"O trabalho em equipe é o trabalho que se compartilha, negociando-se as distintas necessidades de decisões técnicas, uma vez que seus saberes operantes particulares levam a bases distintas de julgamentos e de tomada de decisões quanto a assistência ou cuidados a se prestar" 11 (p. 233).

Pires 14 (p. 91) defende a posição de que "é preciso pensar e propor formas de organização do trabalho que tenham um impacto na qualidade da assistência e, ao mesmo tempo, considerem a possibilidade da realização de um trabalho interdisciplinar, criativo e integrador da riqueza da diversidade de formação dos profissionais da saúde. Um trabalho que possibilite a satisfação pessoal e a integração do potencial humano do trabalhador, com vistas a superar a realidade majoritária do locus do trabalho com deformidade".

\section{Conclusões}

Procurou-se ressaltar as condições vigentes na implementação da atual proposta de trabalho no PSF, do governo brasileiro, conduzido em um contexto neoliberal e fortemente influenciado pelo modelo biomédico e pela lógica taylorista de divisão e organização do trabalho. Os profissionais e as instituições necessitam ter clareza das condições vigentes, uma vez que as possibilidades de sucesso e fracasso não são exclusivas de seus desempenhos, mas também de características próprias dos serviços de saúde e das determinações histórico-estruturais.

Vimos que as condições de trabalho em saúde se deterioram no Brasil, a partir do final da década de 80 , pela influência da política neoliberal e em virtude do aumento das demandas 
em saúde de uma grande parcela da população empobrecida e desprotegida socialmente, ao mesmo tempo em que o setor de saúde é submetido a rígida contenção de custos.

A construção de um novo modelo assistencial em saúde, no sentido dos valores propostos pelo SUS e que aparecem, em parte, no PSF, é um desafio. É essencial um modelo de organização dos serviços de saúde alicerçado em condições sócio-políticas, materiais e humanas, que viabilize um trabalho de qualidade para quem o exerce e para quem recebe a assistência. A não-realização de um trabalho focado nesses dois olhares, no PSF, corre o risco de fazer ruir a motivação e os créditos dados pelos profissionais da saúde e pela sociedade à referida proposta. É fundamental que as contradições e dificuldades que permeiam a proposta não a levem a destruir-se, e, sim, a modificar-se no sentido de melhor qualificação.

\section{Resumo}

O objetivo desta revisão bibliográfica foi destacar a utilidade da teorização sobre o processo de trabalho em saúde para analisar a realidade cotidiana do Programa Saúde da Família (PSF). Desde 1994, o PSF vem rapidamente expandindo a cobertura em saúde da população brasileira e conseqüentemente sua força de trabalho. Assim demanda atenção, responsabilização e contribuições de várias naturezas, de atores e instituições. Trata-se de um trabalho do setor de serviços que ocorre em uma conjuntura neoliberal.Compartilha características de outros trabalhos do setor de serviços, como a burocratização, a influência da divisão parcelar do trabalho e as dificuldades do trabalho em equipe, além da pouca consideração da subjetividade de usuários e trabalhadores. Destacam-se condições do trabalho das equipes de saúde da família, alternativas para revitalização do trabalho em saúde e enfrentamento de condições alienantes do processo de trabalho. Finalmente, ratifica-se a visão da condição dialética do processo de trabalho em saúde no PSF, com suas possibilidades de sucesso, concebido e funcionando permeado por contradições e dificuldades.

Programa Saúde da Família; Condições de Trabalho; Trabalhadores
Há concretizações e sonhos de novas pautas de conduta, de revitalização da prática de sujeitos ética, politica e socialmente diferenciados, de novas parcerias e de mudanças institucionais, perpassando o PSF.

Por outro lado e conforme Bertoncini 17 (p. 67), “as equipes saúde da família transitam num ideário permeado de contradições,espaço de luta no qual os vários projetos dos atores sociais em situação divergem e convergem". Nos diversos depoimentos das equipes de saúde da família, evidenciam-se os conflitos decorrentes da posição dos atores e de serviços na arena de construção de um novo modelo de produção da saúde, em face da lógica e condições do processo de trabalho em que se inserem.

A tarefa é desafiadora, já que requer rompimentos, enfrentamentos, criação, opção, ação nas macro, intermediária e micro dimensões.

\section{Colaboradores}

Uma primeira versão do trabalho foi elaborada por E. M. Ribeiro. D. Pires e V. L. G. Blank participaram da reformulação do manuscrito. 


\section{Referências}

1. Ministério da Saúde. PSF: quantos somos. http:// www.gov.saúde.gov.br/programa/index.asp (acessado em 10/Abr/2003).

2. Braverman H. Trabalho e capital monopolista: a degradação do trabalho no século XX. Rio de Janeiro: Zahar; 1981.

3. Mils W. A nova classe média. Rio de Janeiro: Zahar; 1979.

4. Offe C. Trabalho e sociedade. Rio de Janeiro: Tempo Brasileiro; 1991.

5. Pires D. O debate teórico sobre trabalho no setor de serviços. Campinas: Universidade Estadual de Campinas; 1994.

6. Pires D. Reestruturação produtiva e trabalho em saúde no Brasil. São Paulo: AnnaBlume; 1998.

7. Pires D. Reestruturação produtiva e conseqüências para o trabalho em saúde. Rev Bras Enfermagem 2000; 53:251-63.

8. Campos GWS. Subjetividade e administração de pessoal: considerações sobre modos de gerenciar o trabalho em equipes de saúde. In: Merhy EE, Onocko RT, organizadores. Agir em saúde um desafio para o público. São Paulo: Editora Hucitec; 1997. p. 229-66.

9. Merhy EE. O ato de governar as tensões constitutivas do agir em saúde como desafio permanente de algumas estratégias. Ciênc Saúde Coletiva 1999; 4:305-29.

10. Sá MC. O mal-estar nas organizações de saúde: planejamento e gestão como problemas ou soluções? Ciênc Saúde Coletiva 1999; 4:255-8.

11. Schraiber LB, Peduzz IM, Sala A, Nemes MIB, Castanheira ERL, Kon R. Planejamento, gestão e avaliação em saúde: identificando problemas. Ciênc Saúde Coletiva 1999; 4:221-42.

12. Soares LT. As atuais políticas de saúde: o risco do desmonte neoliberal. Rev Bras Enfermagem 2000; 53:17-24.

13. Almeida MCP, Rocha JSY. O saber da enfermagem e sua dimensão prática. São Paulo: Cortez; 1986.

14. Pires D. Novas formas de organização do trabalho em saúde e enfermagem. Rev Baiana Enfermagem 2000; 13:83-92.
15. Mattos RA. Em busca de novos projetos coletivos. Ciênc Saúde Coletiva 1999; 4:250-2.

16. Ministério da Saúde. Saúde da família: uma estratégia para a reorientação do modelo assistencial. Brasília: Coordenação de Saúde da Comunidade, Secretaria de Assistência à Saúde, Ministério da Saúde; 1997.

17. Bertoncini JH. Da intenção ao gesto - uma análise da implantação do Programa de Saúde da Família em Blumenau [Dissertação de Mestrado]. Florianópolis: Departamento de Saúde Pública, Universidade Federal de Santa Catarina; 2000.

18. Sousa MF. A Cor-Agem do PSF. São Paulo: Editora Hucitec; 2001.

19. Fertomani HP. O desafio de construir um novo modelo assistencial em saúde: reflexões de trabalhadores do PSF de uma unidade de saúde de Maringá-PR [Projeto de Qualificação de Dissertação de Mestrado]. Florianópolis: Departamento de Enfermagem, Universidade Federal de Santa Catarina; 2003.

20. Costa ARC, Melo MC, Oliveira MA, Dias RN. Atuação do enfermeiro no Programa de Saúde da Família em Sobradinho II. Rev Bras Enfermagem 2000; 53:127-30.

21. Costa MBS, Lima CB, Oliveira CP. Atuação do enfermeiro no Programa de Saúde da Família (PSF) no Estado da Paraíba. Rev Bras Enfermagem 2000; 53:149-52.

22. Souza HM. Programa de saúde da família. Rev Bras Enfermagem 2000; 53:7-16.

23. Franco T, Merhy EE. PSF: contradições e novos desafios. htpp://www.datasus.gov.br/cns/temas/ Tribu-buna/PSFTito.html (acessado em 20/Abr/ 2002).

24. Azevedo CS, Braga Neto FC, Sá MC. Indivíduos e a mudança nas organizações de saúde: contribuições da psicossociologia. Cad Saúde Pública 2002; 18:235-247.

Recebido em 03/Set/2002

Versão final reapresentada em 28/Mar/2003 Aprovado em 16/Out/2002 